\title{
Asistencia temprana a Salas Cuna y patrones de apego infantil: Una revisión ${ }^{1}$
}

\section{Early assistance to the child care centers and patterns of infant attachment: A review}

\author{
María Pía Santelices ${ }^{2}$ y Marcia Olhaberry \\ Pontificia Universidad Católica de Chile
}

(Recepción: Diciembre 2008 - Aceptación: Abril 2009)

\begin{abstract}
Resumen
El presente artículo busca reflexionar acerca de la influencia de los cuidados alternativos en la vinculación madre-hijo. Se estudian los hallazgos de investigaciones, los cuales son controversiales, no permitendo establecer un tipo de influencia específica en la asistencia a Centros de Cuidado Infantil durante el primer año de vida. Se revisan investigaciones desde 1978 hasta el 2008 acerca de los cuidados alternativos tempranos y su efecto en los estilos de apego infantil. Se revisan principalmente los estudios referidos a cuidados fuera del hogar en Centros especializados. Se desarrolla la definición de cuidados alternativos, la importancia del apego y la función de los cuidadores como base segura. Se revisan investigaciones centradas en la cantidad de tiempo en que los niños son cuidados por alguien distinto de la madre, la calidad de los cuidados y las variables asociadas a la familia y el contexto social. Esto permite una mirada más completa y compleja del efecto de los cuidados alternativos en el desarrollo de patrones de apego infantil. Se discuten los distintos hallazgos y se destacan aquellos de mayor consistencia que pudieran contribuir a entender el discutido efecto de los Centros de cuidado infantil y su relación con los patrones de apego infantil.

Palabras clave: cuidados alternativos, apego infantil, centros de cuidado infantil.
\end{abstract}

\begin{abstract}
This paper reflects on the influence of alternative care in the mother-child attachment. We study the controversial findings that do not allow the kind of influence of assistance to Child Care Centers in the first year of life. We review research from 1978 to 2008 on alternative care early and their effect on infant attachment patterns. We review the studies referred mainly to out-of-home care in specialized centers. It develops the definition of alternative care, the importance of adherence and the role of caregivers as a secure base. We review research that focuses on the amount of time in which children are cared for by someone other than the mother, the quality of care and the variables associated with the family and social context. This allows a more comprehensive and complex the effect of alternative care in the development of infant attachment patterns. It discusses the various findings and highlights those of consistency that could contribute to understanding the discussion of the effect of child care centers and their relation to patterns of infant attachment.
\end{abstract}

Keywords: alternative care, child attachment, child care centers.

1 La elaboración de este artículo contó con el financiamiento otorgado por el Fondo Nacional de Desarrollo Científico y Tecnológico FONDECYT No 1070839.

2 Correspondencia a: María Pía Santelices, Escuela de Psicología, Pontificia Universidad Católica de Chile. Av. Vicuña Mackenna 4860, Santiago de Chile. Teléfono: 3547664. Correo electrónico: msanteli@uc.cl 


\section{Introducción}

La vida moderna y la creciente incorporación de la mujer al mundo del trabajo, han generado que muchos niños(as) pequeños reciban tempranamente cuidados diarios alternativos brindados por adultos distintos de la madre. El estudio del efecto de los cuidados alternativos en los niños tiene una larga data, y se ha vuelto de gran importancia por la gran frecuencia con que actualmente niños pequeños asisten a centros de cuidado mientras sus madres trabajan. Friedman \& Boyle, (2008) definen cuidados alternativos como todas aquellas rutinas de cuidados diarios realizadas por cualquier adulto distinto de la madre, lo que incluye al padre, abuelos, nanas, otros familiares y personal de Centros de Cuidado.

En Chile, el ingreso de niños pequeños a salas cuna tiene muchas veces una motivación centrada en la necesidad de la madre y/o del grupo familiar de incorporación al trabajo. Por otro lado a partir del aumento de la cobertura para la atención de niños menores de 3 años en los centros de cuidado en Chile, que buscan igualar las oportunidades de desarrollo integral para los niños pertenecientes a los sectores más pobres de la población y los antecedentes que señalan el valor protector del logro de apego seguro entre los niños y sus padres, surge la necesidad de revisar los estudios existentes en relación al efecto de los cuidados institucionales en los patrones de apego que los niños desarrollan.

Contar con la opción de cuidados alternativos como salas cuna o jardines infantiles pudiera favorecer en madres y familias que viven en condiciones de alto estrés psi-cosocial, una mejora en la calidad vincular con sus hijos a partir de la vivencia de mayor apoyo. En este sentido los planteamientos de Crittenden, (1985), pudieran respaldar esta idea, al señalar la existencia de una relación positiva entre adecuadas redes de apoyo para la madre y menor frecuencia de maltrato y negligencia hacia los hijos, pudiendo reconocerse en los centros de cuidado una red de soporte familiar.

Por otro lado estudios recientes realizados por Green, Ferrer \& McAllister, (2007) señalan que los padres que cuentan con mayores redes de apoyo mejoran la calidad de las interacciones con sus hijos, observándose esto principalmente en padres con patrón de apego ansioso y no en los padres con patrón evitativo. Esto permite pensar que probablemente el apoyo que implican los centros de cuidado infantil para los niños, pudieran tener efectos diferenciales dependiendo del estilo de apego de los padres. En este contexto, el presente artículo pretende hacer una revisión acerca de los efectos del cuidado infantil alternativo ene $l$ apego infantil.

\section{Estilos de apego infantil e implicancias en el desarrollo}

John Bowlby, desarrolla la Teoría del Apego, como una forma de explicar el desarrollo afectivo humano. Señala que la necesidad de establecer vínculos estables con los progenitores (o sustitutos) es una necesidad primaria, no aprendida, propia de la especie humana (Bowlby, 1958). Esta tendencia a desarrollar fuertes lazos afectivos se expresa en conductas desarrolladas inicialmente en la infancia y posteriormente en la adultez, para lograr proximidad con las figuras de apego, identificadas como mejor capacitadas para enfrentarse al mundo, en los momentos de ansiedad, temor o estrés (Bowlby, 1969,1980, 1995, 1997). A partir de estos supuestos distintos autores plantean que la seguridad en el apego desarrollada entre el niño y sus progenitores actuaría como un factor protector en el desarrollo infantil, así como la inseguridad del apego sería un factor de riesgo (Greenberg, 1999).

Los patrones de apego infantil definidos por Mary Ainsworth a partir del procedimiento de la situación extraña, son los patrones seguro, ansioso ambivalente y evitativo (Ainsworth, Blehar, Waters \& Wall, 1978).

Los niños(as) con apego seguro logran explorar su entorno a partir de la percepción de su madre como una base segura. Recienten la separación de ella y buscan acercarse y ser consolados en el re-encuentro. 
Los niños con apego ansioso ambivalente responden frente a la separación con una intensa angustia, alternando conductas de apego con enojo y resistencia al momento del re-encuentro. No confían en la respuesta protectora y oportuna del cuidador, ya que su experiencia es de un cuidador inconsistente, lo que les dificulta poder tener una expectativa clara de su conducta. Presentan dificultades para regular adecuadamente sus emociones tendiendo a amplificarlas.

Los niños con apego evitativo se muestran muy independientes frente a las separaciones y las vivencias de amenaza, evitando la cercanía. Exploran libremente su entorno sin recurrir a la madre frente a la dificultad el estrés o el temor. Rechazan el contacto con la madre al momento del reencuentro o manifiestan poco interés en ella. Sobre-regulan sus emociones mostrando indiferencia y calma a pesar de experimentar tensión.

Un cuarto patrón de apego desarrollado por Main y Salomón en 1986 (citado en Marrone, 2001), es el patrón de apego desorganizado. Fue desarrollado con posterioridad, buscando clasificar aquellos niños(as), que no podían ser reconocidos en los tres patrones ya descritos por Ainsworth. Muestran características de los patrones inseguros ansioso/ambivalente y evitativo, pero de manera alternada. Actúan de manera confusa, dando mensajes contradictorios, que hacen difícil interpretar su comportamiento.

En relación a las implicancias en la salud mental infantil, los patrones de apego seguro permiten predecir en los niños un desarrollo saludable, autoestima positiva, identidad integrada, éxito educacional y mayor capacidad para enfrentar situaciones de estrés (Fonagy, 1999). Se observa también en niños con apego seguro una mayor resistencia emocional, elevada auto-confianza, adecuado desarrollo social, un mayor repertorio empatito y habilidades para establecer relaciones interpersonales más profundas (Waters, Wippman \& Sroufe, 1979; Sroufe, Egeland \& Kreutzer, 1990), constituyéndose el apego seguro en un elemento protector en el desarrollo y promotor de salud mental infantil.

Los distintos estudios en cuidados alternativos se han centrado principalmente en los tres patrones definidos por Ainsworth y han usado mayoritariamente la situación extraña como medida de evaluación del apego infantil. Se ha criticado el uso de este procedimiento para evaluar apego en niños que asisten a centros de cuidado, por pensarse que el acostumbramiento a vivir separaciones prolongadas diariamente pudiera no ser suficiente para activar el sistema de apego. Friedman \& Boyle, (2008) señalan que la situación extraña si sería un buen procedimiento para evaluar apego en niños que tienen jornadas extensas de cuidados alternativos. Se basan para esta afirmación en los estudios realizados en kibbutz con niños con 50 horas semanales de cuidados alternativos que mostraban altos niveles de estrés en la separación de sus madres y alta activación del sistema de apego, desmintiendo la idea de acostumbramiento frente a separaciones frecuentes y prolongadas.

\section{Cuidados alternativos}

El debate en torno a los efectos de los distintos tipos de cuidados alternativos recibidos por los niños en su primer año de desarrollo, en la relación de apego hacia su madre cuenta con una larga trayectoria, con importantes discrepancias.

Los distintos estudios realizados sobre cuidados alternativos y su asociación con los estilos de apego infantil se han centrado en distintos aspectos, algunos en la extensión horaria y el lugar donde los cuidados ocurren, otros en la calidad de los cuidados recibidos, en la edad en que se inician y los más actuales consideran múltiples variables además del apego y los cuidados brindados por alguien distinto de la madre para explicar el vínculo madre-hijo(a).

Inicialmente a partir de los estudios de Belsky \& Steinberg (1978), se planteó que la asistencia de los niños pequeños a centros de cuidado de alta calidad no tenía efectos negativos en su desarrollo intelectual, no interfería el vínculo emocional con su madre y aumentaba el grado en que los niños interactuaban positiva y negativamente con sus pares. 
Estos resultados reforzaron la idea del valor de la asistencia a la sala cuna y al jardín infantil como espacios favorables para aprender cosas nuevas que el contexto de la casa no favorece y para contribuir al desarrollo de habilidades sociales al compartir con pares.

Posteriormente en 1988, Belsky realiza nuevos estudios, encontrando que la asistencia a centros de cuidado antes del año genera en el niño una vulnerabilidad socio-emocional que pudiera predisponer al desarrollo de un patrón de apego inseguro hacia la madre, contradiciendo los hallazgos de su estudio anterior. Estos estudios reflejan históricas controversias sobre cuidados alternativos que los estudios actuales buscan esclarecer.

\section{La importancia de la extensión horaria en los cuidados alternativos}

En relación al tiempo semanal con cuidados alternativos, los estudios realizados por Schwartz, (1983) en niños que asistían a centros de cuidado antes de los 9 meses en jornada completa, observó con mayor frecuencia conductas evitativas en los niños en el episodio final de la situación extraña, no así en los niños que asistían tiempo parcial. Posteriormente el meta-análisis realizado por Belsky y Rovine en 1988 confirman los hallazgos de Schwartz encontrando que una gran extensión horaria (20 horas o más) de cuidados alternativos para los niños(as) durante su primer año de vida constituye un factor de riesgo para el desarrollo de apegos inseguros, principalmente evitativos, no observándose esta relación cuando la asistencia es menor a 20 horas. Contrario a estos hallazgos son los resultados obtenidos por Belsky \& Braungart (1991), quienes evaluaron sólo niños con apego evitativo y jornadas de 20 horas o menos y más de 20 horas, encontrando que la jornada extensa se asociaba a llanto y protestas más intensas en los niños y no a sobre- regulación de sus emociones frente a la separación como se esperaría en el patrón de apego evitativo. Estos estudios señalan además que los cuidados alternativos que más parecían lesionar la formación de un vínculo seguro con la madre, además de los que implicaban un mayor número de horas, eran los que ocurrían fuera de la casa en Centros de atención. Al analizar a los niños que presentaban apegos seguros y un tiempo mayor a 20 horas de cuidado destacan la importancia de otras variables como la adecuada sensibilidad materna, la satisfacción marital en la madre, la presencia de padres juntos, la percepción del niño por parte de la madre y el estilo de apego del niño desarrollado con el padre. Lo anterior apoya la idea de que un alto número de horas de cuidados alternativos recibidos por los niños en su primer año de vida pudiera constituirse en un "factor de riesgo" para el desarrollo de apegos inseguros, que pudiera o no desarrollarse de acuerdo a las variables familiares señaladas.

Estudios realizados por Barglow, Vaughn \& Monitor, (1987) evaluando el efecto de los cuidados alternativos antes del año realizados en la casa en el patrón de apego infantil, los hallazgos son semejantes, encontrando que el que la madre esté ausente jornada completa por su trabajo constituye un factor de riesgo para el desarrollo de apegos inseguros evitativos en los niños no así cuando la madre se ausenta media jornada.

Estudios posteriores realizados por Hoffman (1989), contradicen estos hallazgos y señalan una relación débil entre cuidados alternativos durante el primer año y el estilo de apego desarrollado por el niño(a), explicando la inseguridad del apego a partir de variables familiares más que por el hecho de recibir cuidaos por personas ajenas al grupo familiar. Roggman et al (1994) critican los meta-análisis realizados por Belsky y Rovine, por considerar que no incluyeron en sus análisis estudios no publicados que no encontraron relación entre mayor tiempo de cuidados alternativos y apegos inseguros. Buscan testear nuevamente la importancia del número de horas en que los niños reciben cuidados alternativos en la semana, estudiando tres grupos, tiempo total, parcial (entre $10 \mathrm{y}$ 20 horas) y sin cuidados alternativos. Encontraron que los niños que presentaban apegos inseguros con mayor frecuencia eran los que asistían por tiempo parcial, explicándose estos resultados a partir de la mayor ansiedad y tensión en las madres que deben cumplir simultáneamente roles maternales y profesionales. Estos resultados nos llevan a reflexionar sobre el peso del estilo vincular de las madres, su relación con el trabajo y las representaciones en torno a la asistencia a sala cuna o jardín infantil como variables influyentes en el tipo de interacción con los hijos(as) y el posterior patrón de apego que estos desarrollan. 


\section{La importancia del momento en que se inician los cuidados alternativos}

Los estudios realizados por Vaughn, Gove \& Egeland (1980), analizan la importancia del momento en que los niños inician su asistencia a centros de cuidado y el efecto en el patrón de apego hacia la madre en familias de nivel socioeconómico bajo. Encontraron que los niños que reciben cuidados alternativos en Centros especializados antes del año de edad muestran en mayor proporción apegos ansiosos ambivalentes hacia sus madres, que los niños que asisten después del año o son cuidados por sus madres. Estos antecedentes destacan que los niños pueden no perjudicar su desarrollo y calidad vincular con la madre si la asistencia a Centros de Cuidado ocurre más allá del año de edad, probablemente por tener el niño mayores recursos afectivos y cognitivos para comprender la experiencia, así como mayor tiempo de interacción cotidiana con su madre que le permite tener mayor claridad en relación a que esperar de ella en relación a su disponibilidad como base segura y confiable. Por otro lado incluyeron sólo familias en pobreza, donde probablemente sea necesario considerar otras variables contextuales que pudieran incidir en los resultados mas allá de la edad de ingreso, como pudieran ser la configuración familiar, depresión en la madre, sensibilidad materna y nivel educacional de los padres, variables influyentes en la calidad vincular madre-hijo.

\section{La importancia de la calidad en los centros de cuidados alternativos}

Otro aspecto relevante en la consideración del impacto de los cuidados alternativos en los niños, es la calidad de la atención que reciben, dentro de la que se puede considerar el número de niños a cargo por adulto, el entrenamiento y preparación con que los profesionales cuentan, la infraestructura en donde se trabaja, así como la salud mental y la estabilidad laboral de los cuidadores. En este sentido destacan los estudios longitudinales de Andersson $(1989,1992)$ realizados en Suecia, donde encontró que los niños que recibieron cuidados alternativos durante el primer año, son evaluados por sus profesores en la etapa escolar (a los 8 y a los 13 años) como más competentes social, emocional y cognitivamente que los niños cuidados exclusivamente por sus madres. Por otro lado encontró también que la asistencia a un centro de cuidado durante el primer año de vida podía mediar el efecto del status socioeconómico en el desarrollo de las habilidades mencionadas a los 8 y a los 13 años. Estos resultados no concuerdan con los de Belsky, pudiendo también existir variables culturales y políticas públicas particulares que expliquen las diferencias entre población norteamericana y sueca, a partir de una alta calidad en los centros de cuidado suecos. Los estudios de Howes (1990), apuntan en esta misma dirección, señalando que lo que mejor predice adaptación posterior en los niños es la calidad del Centro de cuidados al que asistieron durante el primer año.

Por otro lado no siempre los niños que presentan apegos inseguros muestran problemas emocionales en la etapa pre-escolar, probablemente por que en algunas ocasiones la asistencia de los niños pequeños a sala cuna puede aliviar a la madre de algunas de sus responsabilidades en el cuidado infantil, influyendo positivamente en su bienestar emocional, en su capacidad de responder de manera sensible, mejorando la calidad de la vinculación. Los estudios de Egeland y Hiester (1995) respaldan esta idea, encontrando que los niños cuidados por sus padres que presentan apego seguro a los 12 meses muestran adecuada adaptación emocional en la etapa pre-escolar y los que presentaban apegos inseguros no, pero en los niños que recibieron cuidados alternativos esta relación no se observa, encontrándose niños con apegos inseguros y buena adaptación posterior. En relación al efecto de la asistencia a un centro de cuidados, este era negativo en los niños con apego seguro y positivo en los niños con apego inseguro en relación a la adaptación socio-emocional a los 3 años y medio. Esto nos lleva a reflexionar si la adaptación escolar posterior es una buena medida de la calidad vincular entre padres e hijos o pudiera también encontrarse en niños que sobre-regulan sus emociones y presentan probablemente apegos inseguros evitativos. Otra lectura que pudiera hacerse es que a pesar de tener apegos inseguros el efecto positivo de los Centros de cuidado favorece su adaptación escolar posterior aunque el patrón de apego con la madre no cambie.

\section{Diferencias en los tipos de cuidados alternativos}

Estudios realizados por Sagi et al (2002) muestran que los niños que asisten durante el primer año a Centros de cuidados llegan a desarrollar más probablemente apegos inseguros hacia sus madres 
al año de edad, que los que son cuidados por su madre, por un cuidador individual no familiar pagado o por otro familiar. Observaron además que la mayoría de los niños desarrollaban apegos inseguros ambivalentes y sólo unos pocos apegos evitativos, siendo estos hallazgos consistentes con los encontrados en estudios previos desarrollados por Scher \& Mayseles (2000) y Van Ijzendorm \& Sagi (1999).

Los estudios desarrollados por Love et al (2003), muestran que la calidad de los centros es el mejor predictor del tipo de apego que los niños pudieran desarrollar con su madre, ejerciendo además la calidad un efecto moderador sobre el tiempo que los niños reciben cuidados alternativos, a más calidad menor impacto negativo del tiempo.

Dentro de las variables consideradas en la influencia de la asistencia a centros de cuidados de los niños antes del año de vida en el estilo de apego a la madre que desarrollan el primer año, la sensibilidad materna ha sido evaluada como un aspecto importante (De Wolf \& Van Ijzendoorn 1997). Estudios realizados por Avizier, Sagi- Schuartz \& Koren-Karie 2003, cuestionan la relación entre adecuada sensibilidad materna y apego seguro en el niño, ya que esta asociación se observa sólo en los niños que tienen un cuidador individual, pero en los que asisten a centros de cuidado y comparten cuidador se observan en igual proporción apegos inseguros en los niños con madres sensibles e insensibles. Estos hallazgos cuestionan el valor predictivo de la sensibilidad materna en relación a la seguridad del apego, y plantean que el compartir cuidador con otros niños en el centro de cuidados pudiera moderar la relación con la madre. El meta-análisis desarrollado por Wolf \& Van Ijzendorm, (1997), señala que existe una relación entre sensibilidad materna y el estilo de apego que los niños desarrollan, pero no sería una condición exclusiva. Se explican esto a partir de que la mayoría de los estudios son hechos en familias de clase media o población no clínica, donde la relación entre respuesta sensible materna y apego seguro en los niños es mayor, pero en familias pobres, el peso de las variables contextuales pudiera interferir esta relación y requiere ser estudiado.

Para comprender estas diferencias resulta importante considerar también la calidad de la vinculación entre el niño y su cuidadora, pudiendo hipotetizarse que una relación positiva con la cuidadora pudiera compensar la relación deficitaria con la madre y esto mejorar la adaptación posterior en la etapa pre-escolar. En este sentido los estudios de Sagi (1990) realizados en niños que asisten a kibbutz, señalan que el mejor predictor de desarrollo emocional de estos niños es el tipo de apego establecido con su cuidadora alternativa.

Diversos estudios han reparado en la importancia de la calidad de los cuidados alternativos, señalando que es el aspecto que en mayor medida se asocia al desarrollo infantil posterior y a su patrón de apego, moderando el efecto del tiempo de duración de estos cuidados (Sagi,2002; Love et al, 2003). La validez metodológica de los estudios de Love 2003 se apoya en que estudió 264 centros de cuidado en USA y en Israel, considerando centros de distintas calidades y definiendo la calidad a partir de lo formal o informal del centro y la existencia o no de regulación de los cuidados.

\section{La importancia de considerar múltiples variables para explicar el efecto de los cuidados alternativos en el estilo de apego desarrollado por los niños}

Se cuestionan los estudios iniciales sobre cuidados alternativos por no considerar otras variables influyentes en el patrón de apego madre-hijo que pudiera ir más allá del tiempo y la calidad y por lo restringido de las muestras desarrollándose nuevas investigaciones para resolver estas limitaciones. En esta línea los estudios desarrollada por el Nacional Institute of Child Health and Human Development (NICHD) Study of Early Child Care and Youth Development (SECCYD) en Estados Unidos (Friedman y Boyle 2008) consideraron la importancia de evaluar otras variables al momento de medir el efecto de la asistencia a centros de cuidado, incluyendo la edad de ingreso, las características del niño, las razones del empleo materno, la satisfacción de la madre en su rol, la relación de pareja y las redes sociales y familiares de apoyo, la sensibilidad materna, la presencia de depresión en la madre y el nivel educacional de los padres entre otros para dar cuenta de la diversidad de aspectos en juego en el efecto de los cuidados alternativos en el apego hacia la madre. 
Destaca el estudio longitudibnal del NICHD por el gran tamaño muestral (1000 niños y su familias) el largo período en que los niños fueron evaluados (desde el nacimiento hasta los 15 años) y la gran diversidad en la muestra (distintos tipos de cuidados alternativos, situación socieconómica, niveles educacionales entre otros). Estos aspectos del estudio dan gran relevancia a sus resultados, donde encontraron correlaciones significativas entre nivel socio económico, el nivel educacional materno, la presencia de depresión materna, ansiedad de separación con la madre y la presencia de los dos padres en la familia con el estilo de apego desarrollado por los niños. Al realizar regresiones logísticas la sensibilidad materna a los 6 y a los 15 meses es la única variable que permite predecir apego seguro a los 15 meses. A su vez también encontraron que la depresión materna interactúa con la sensibilidad materna y predice inseguridad en el apego infantil.

Quizás los hallazgos más sorprendentes son los referidos a los efectos de los cuidados alternativos en el patrón de apego infantil, señalando que la edad de inicio de los cuidados alternativos, la estabilidad de los cuidados, el tipo de cuidados y la calidad de estos no tienen un efecto sobre el apego desarrollado por el niño hacia la madre.

\section{Discusión y conclusiones}

Si bien el respaldo metodológico del estudio del NICHD es de gran fuerza, cabe reflexionar si el 9\% de la muestra que asistió a Centros de cuidado es suficiente para afirmar que las variables asociadas a extensión, calidad y edad de ingreso no tendrían ningún efecto sobre el estilo de apego infantil. Resulta difícil pensar que la calidad del cuidador sustituto no tenga ningún efecto sobre el patrón de apego de un niño menor de un año que asiste a sala cuna en jornada extensa, ya que probablemente ese niño comparte un gran número de horas a la semana con el cuidador alternativo, explicándose esto tal vez por que la influencia de esta relación pudiera no apreciarse en el patrón de apego sino en otras variables. Si pensamos en el niño y el cuidador como díada que se influye mutuamente, probablemente sea necesario estudiar variables más sutiles que están presentes en el patrón de apego del niño para evaluar si el intercambio con el cuidador sustituto genera alguna modificación positiva o negativa en el estilo vincular del niño, que no se observa directamente en el patrón general.

Por otro lado es posible también pensar que los patrones de apego se establecen en relación a los padres o a los cuidadores primarios, por lo que niños que asisten a sala cuna por jornadas extensas y que cuentan con sus padres de manera estable desde el nacimiento, puedan tener interacciones afectivamente significativas con otros adultos pero que no se constituyan en figuras de apego. Esto lleva a cuestionarse de que manera un adulto se constituye como figura de apego, si por el tiempo compartido y las vivencias emocionales positivas y negativas, o por la permanencia de la vinculación, que aunque positiva o negativa es reconocida por el niño como estable.

Otra explicación posible para entender la ausencia de efecto de las variables asociadas a los cuidados alternativos en el patrón de apego desarrollado en los niños, podría estar en que probablemente las madres y padres toman decisiones sobre los cuidados alternativos para sus hijos en virtud de sus propios modelos operativos internos. Esto implicaría que la madre más rechazante probablemente considere el ingreso temprano a la sala cuna o el destete precoz como una buena opción, sin considerar los aspectos emocionales que esta decisión pudiera implicar para el niño. La madre segura con mayor probabilidad pudiera buscar opciones de cuidado que incluyan en bienestar emocional, y tener mayor conciencia de las necesidades de su hijo y la madre ansiosa tal vez explore en muchas alternativas diferentes y no logre generar una opción estable, generando cada una desde su estilo vincular las condiciones que tienden a fortalecer el patrón de apego establecido con sus hijos.

Por otro lado resulta relevante pensar de que manera puede influir un centro de cuidados alternativos de calidad en niños con patrones de apego inseguro con sus padres, pudiendo tal vez tener un efecto favorable. Los estudios de Egeland \& Hiester, 1995 que señalan que los niños evitativos muestran cambios positivos en la asistencia a centros de cuidados, pero no así los seguros, 
respaldan esta idea. Si pensamos en como pueden cambiar los patrones de apego en los adultos, surge la psicoterapia como una posible experiencia emocional correctiva que permite modificar modelos representacionales de sí mismo y de los otros, así como relaciones de pareja significativas o la maternidad también pudieran actuar como vínculos correctivos. Al volver a pensar en los niños pequeños, cuesta imaginar que relaciones afectivamente significativas con un cuidador no tengan ningún efecto en el desarrollo vincular infantil. Tal vez algo que podría ayudar a aclarar el punto es evaluar el tipo de apego de los niños que reciben cuidados alternativos con su cuidador sustituto cuando este es permanente y estable en el tiempo, ya que tal vez se da una experiencia correctiva y un apego distinto al observado con la madre, pero que no modifica el estilo vincular desarrollado con su cuidador primario.

Los hallazgos en relación a la respuesta sensible de la madre como un buen predictor de apego seguro (Friedman \& Boyle, 2008), alertan sobre la importancia de considerar esta variable al diseñar programas de intervención familiar, especialmente cuando estos se dirigen a la familia temprana. Estos hallazgos resultan también consistentes en relación a los múltiples estudios que asocian adecuada respuesta sensible materna durante el primer año y apego infantil seguro (BraungartRieker, garwood, Powers \& CNAG, 2001; Coppola, Vaughn, Cassiba, Constantini, 2006; Isabella, 2003; Smith \& Pederson 1988; Thompson, 1997; Van Ijzendoorm \& Wolf 1997; Ward \& Carlson, 1995) Especialmente esperanzador resulta este hallazgo, pensando que la respuesta sensible de los cuidadores puede ser entrenada con éxito a partir de intervenciones focalizadas y en un corto número de sesiones (Bakermans-Kranenburg, Van Ijzendoorm \& Buffer 2003 ).

Es posible que evaluaciones de la respuesta sensible del cuidador alternativo de los centros y la cooperatividad mostrada por el niño en la interacción, pudieran reflejar el efecto de ese intercambio cotidiano, y su cualidad positiva o negativa, pudiendo también observarse si existen diferencias en estas dimensiones en la interacción con la madre. En este sentido tal vez los patrones de apego resultan una medida gruesa de los estilos vinculares que no permiten discriminar modificaciones o diferencias en variables asociadas al apego que más fácilmente pueden cambiar.

Por último otro aspecto a considerar son las variables culturales que pudieran influir y generar diferencias en estudios semejantes al realizado por el NICHD realizados en culturas distintas a la Norteamérica, requiriéndose investigaciones en distintos países para evaluar si estos resultados se repiten.

En síntesis, si bien los hallazgos son múltiples y contradictorios en relación a los centros de cuidado y su efecto en el apego infantil, destacan los estudios longitudinales realizados por el NICHD por su rigurosidad metodológica y amplitud de las variables consideradas. Estos hallazgos señalan la importancia de la adecuada sensibilidad materna como predictor de apego seguro y muestran el poco peso de las características de los cuidados alternativos brindados en Centro de cuidado en el desarrollo de los estilos de apego infantil.

Si los Centros de cuidados alternativos y sus características no pueden ser asociados a estilos de apego particular, tal vez sea relevante invertir la pregunta inicial y plantearse de que manera pudieran los Centros contribuir a mejorar la calidad de los vínculos en niños que presentan apegos inseguros con sus padres. 


\section{Referencias}

Ainsworth, M.D.S., Blehar, M. C., Waters, E., \& Wall, S. (1978). Patterns of attachment: A psychological study of the strange situation. New Jersey: Eribaum.

Andersson B. E. (1989). Effects of Public Day-Care: A Longitudinal Study. Child Development, 60, (4), 857-866.

Andersson B. E. (1992). Effects of Day-Care on Cognitive and Socioemotional Competence of Thirteen-Year-Old Swedish Schoolchildren. Child Development, 63, (1), 20-36.

Aviezer, O. \& Sagi-Schwartz A. (2008). Attachment and non-maternal care: towards contextualizing the quantity versus quality debate. Attachment and Human Development, 10 (3), 275-285.

Aviezer O., Sagi-Schwartz A. \& Koren-Karie N. (2003). Ecological constraints on the formation of infant-mother attachment relations: When maternal sensitivity becames ineffectives. Infant Behavior and Development, 26, 285-299.

Bakermans-Kranenburg M., Ijzendoorn M. \& Juffer F. (2003). Less is More: Meta-Analyses of Sensitivity and Attachment Interventions in early Childhood. Psychological Bulletin,129(2), 195-215.

Barglow P., Vaughn B. \& Molitor N. (1987). Effects of Maternal Absence Due to Employment on the Quality of Infant-Mother Attachment in a Low-Risk Sample. Child Development, 58, (4), 945-954.

Belsky J. \& Braungart J. (1991). Are Insecure-Avoidant Infants with Extensive Day-Care Experience Less Stressed by and More Independent in the Strange Situation?. Child Development ,62,(3), 567-571.

Belsky J. \& Rovine M.J. (1988). Nonmaternal Care in the First Year of Life and the Security of Infant-Parent Attachment. Child Development, 59, (1), 157-167.

Belsky J. \& Steinberg L. (1978). The Effects of day-care A critical review. Child Development, 49, 929-949.

Bowlby, J. (1958). The nature of the child's tie to his mother. Internacional Journal of PsychoAnalysis, 39,350-373.

Bowlby, J. (1969). El vínculo afectivo. Buenos Aires: Paidós.

Bowlby, J. (1976). La separación afectiva. Buenos Aires: Paidós

Bowlby, J. (1980). La pérdida afectiva. Buenos Aires: Paidós.

Bowlby, J.(1995). Una base segura. España: Paidós.

Bowlby, J.(1997). El vínculo afectivo. España: Paidós.

Braungart-Rieker J., Garwood M., Powers B. \& Wang X. (2001). Parental Sensitivity, Infant Affect, and Affect Regulation: Predictors of Later Attachment. Child Development, 72(1), 252-270.

Coppola, G., Vaughn B., Cassiba R. \& Costantini A. (2006). The attachment script representation procedure in an Italian simple: Associations with Adult Attachment Interview scales and with maternal sensitivity. Attachment \& Human Development 8(3): 209-219.

Crittenden, P. (1985). Social Networks, Quality of Child Rearing and Child Development. Child Development, 56, 1299-1313.

De Wolff M. \& Van Ijzendoorn M. (1997). Sensitivity and Attachment: A Meta-Analysis on Parental Antecedents of Infant Attachment. Child Development, 68 (4), 571-591. 
Egeland B. \& Hiester M. (1995). The long-term consequences of infant day-care and mother-infant attachment. Child Development, 66, (2), 474-485.

Fonagy, P. (1999). Persistencias transgeneracionales del apego: Una nueva teoría. Revista de Psicoanálisis, Aperturas Psicoanalíticas, 3, 17-25.

Friedman S. \& Boyle D.E. (2008). Attachment in US children experiencing nonmaternal care in the early 1990s. Attacment and Human Development, 10 (3), 225-261

Green B., Ferrer C. \& McAllister C. (2007). How do relationships Support Parenting? Effects of Attachment Style and Social Support on Parenting Behavior in an At-Risk Population. American Journal of Community Psychology, 40, 96-108.

Greenberg M. (1999). Attachment and Psychopathology in Childhood. En J. Cassidy \& P.R. Shaver (Eds.), Hanbook of Attachment: Theory, research and clinical applications (pp. 469-496). New York: Guilford Press.

Hoffman L.W. (1989). Effects of maternal employment in the two-parent family. American Psychologist, 44, 283-292.

Howes C. (1990). Can the age of entry and the quality of infant child care predict adjustment in kindergarten?. Developmental Psychology, 26, 292, 303.

Isabella R.A. (1993). Origins of attachment: maternal interactive behavior across the first year. Child Development, 64, 605-621.

Love J.M., Harrison L., Sagi-Schwartz A., van Ijzendoorn M.H., Ungerer J.A. (2003). Child care quality matters: How conclusions may vary with context. Child Development, 74, 1021-1033.

Marrone, M.(2001). La teoría del apego. Un enfoque actual. Madrid: Psimática.

Roggman L.A., Langlois J.H., Hubbs-Tait L., Rieser-Danner L.A. (1994). Infant Day-Care, Attachment, and the « File Drawer Problem ». Child Development, 65, (5),1429-1443.

Sagi A. (1990). Attachment theory and research from a cross-cultural perspective. Human Development, 33, 10-22.

Sagi A., Koren-Karie N., Gini M., Ziv Y. \& Joels T. (2002). Shedding Further Light on the Effects of Various Types and Quality of Early Child Care on Infant-Mother Attachment Relationship: The Haifa Study of Early Child Care. Child Development, 73 (4), 1166-1186.

Schwartz P. (1983). Length of Day-Care Attendance and Attachment Behavior in Eighteen MonthOld Infants. Child Development, 54, (4), 1073-1078.

Smith P. \& Pederson D. (1988). Maternal Sensitivity and Patterns of Infant-Mother Attachment. Child Development, 59,1097-1101.

Sroufe, L.A., Egeland, B. \& Kreutzer, T. (1990). The fate of early experience following developmental change: Longitudinal approaches to individual adaptation in childhood. Child Development, 61, 1363-1373.

Thompson R. (1997). Sensitivity and Security: New Questions to Ponder. Child Development, 68(4), 595-597.

Van Ijzendoorn M. \& De Wolf M. (1997). In Search of the Absent Father- Meta-Analyses of InfantFather Attachment: A Rejoinder to Our Discussants. Child Development, 68(4) 604-609.

Vaughn B., Gove F. \& Egeland B. (1980). The Relationships between Out-of-Home Care and the Quality of Infant-Mother Attachment in an Economically Disadvantaged Population. Child Development, 51, (4), 1203-1214. 
Ward M.J. \& Carlson E.A. (1995). Associations among adult attachment representations, maternal sensitivity, and infant-mother attachment in a sample of adolescent mothers. Child Development, 66, 69-79.

Waters, E., Wippman, J. \& Sroufe, L.A. (1979). Attachment, positive affect, and competence in the peer group: Two studies in construct validation. Child Development, 50, 821-829. 